A COMPARISON OF THE MEAN-VALUE AND

\title{
INITIAL-VALUE SOLUTIONS OF THE IDEAL RESONANCE \\ PROBLEM WITH AN APPLICATION IN RIGID-BODY \\ MECHANICS
}

\author{
A. H. JUPP
}

The University of Liverpool, U.K.

\begin{abstract}
A solution of the ideal resonance problem has already been exhibited (Jupp, 1972) explicitly in terms of the 'mean' elements; to second order in the small parameter in the case of libration, and to first order in the case of deep circulation. Both representations possess a singularity when the 'mean' modulus of the Jacobi elliptic functions is unity; this corresponds to motions on or close to the separatrix of the phase plane of the dynamical system.

It is shown that, provided particular coefficients associated with the problem satisfy specific relations, the singularity is removed, and the final solution is applicable throughout the deep resonance region.

The solution is next expressed in terms of the initial conditions of the system. Again, in general, the solution has a singularity associated closely with the limiting motion, and the circulation form of the solution is restricted to deep circulation. It is shown that when the previously-mentioned coefficients satisfy particular constraints, the singularity is removed. Moreover, with the same constraints, the deepcirculation solution extends naturally to cover the entire circulation regime. It is of interest that these constraints are quite different from those associated with the 'mean' element formulation.

In the light of these results a global formal series solution in terms of initial conditions of the problem of the free rotation of a rigid body can simply be extracted from the general solution of the ideal resonance problem. The small parameter is provided by requiring that two of the principal moments of inertia differ by a small quantity. Such a solution can readily be checked with the well-known exact solution.

Tentative conclusions are drawn regarding the removal of the singularity in the two separate formulations, and some interesting aspects of the Lie series perturbation procedures are noted.
\end{abstract}

\section{Reference}

Jupp, A. H.: 1972, Celes. Mech. 5, 8-26.

Y. Kozai (ed.), The Stability of the Solar System and of Small Stellar Systems, 209. All Rights Reserved. Copyright C: 1974 by the IAU. 